American Journal of Animal and Veterinary Sciences 5 (2): 86-90, 2010

ISSN 1557-4555

(C) 2010 Science Publications

\title{
Prevalence of Stray Dogs with Intestinal Protozoan Parasites
}

\author{
Mohammad Mirzaei \\ Department of Pathobiology, Faculty of Veterinary Medicine, \\ Shahid Bahonar University of Kerman, Kerman, Iran
}

\begin{abstract}
Problem statement: Intestinal protozoan parasites are important enteropathogens in dogs. Moreover, several canine intestinal protozoan parasites are zoonotic and are considered important to public health. This study investigates the level of intestinal protozoan parasites in stray dogs, in Kerman city, Iran. Approach: Determination of the prevalence of infections was based on faecal examination. Stool samples $(n=98)$ collected from dogs of different ages and gender were analyzed using five techniques, i.e., centrifugal flotation in sucrose solution, centrifugal flotation in $33 \%$ Zinc solphate solution, Ziehl-Neelsen staining, trichrome staining and iodine staining. Results: The overall prevalence of parasitism was $13(13.26 \%)$ dogs. The parasites most frequently detected were: Giardia spp. (7.14\%), Isospora spp. (5.1\%) and Cryptosporidium spp. (4.08\%). Single parasitic infection was present in $11(11.22 \%)$ dogs. There was no significant difference in the prevalence between male $(13.3 \%)$ and female $(13.2 \%)$ dogs $(p>0.05)$. There was significantly $(\mathrm{p}<0.05)$ greater prevalence of parasites in dogs less than 1 year old. Conclusion/Recommendations: The results of this research showed that stray dogs are reservoirs for zoonotic intestinal protozoan parasites and should be considered important to public health. So that, it is imperative for human to avoid faecal contamination in streets, public gardens and parks. Also stray dogs should be euthanized in dog population control program in Iran.
\end{abstract}

Key words: Intestinal protozoan parasites, Cryptosporidium, Giardia, Isospora, Stray dogs, Kerman

\section{INTRODUCTION}

Intestinal protozoan parasites are important enteropathogens in dogs. Moreover, several canine intestinal protozoan parasites are zoonotic and are considered important to public health, e.g., Cryptosporidium, Giardia, Entamoeba and Isospora. All these intestinal protozoan parasites have an oralfecal transmission cycle and a major component for the spread of these parasites is the shedding of oocysts or cysts into the environment (Claerebout et al., 2009). It is common to observe intestinal protozoan parasites in canine of all ages, but the prevalence of infection is usually high in puppies, mainly due to the fact that certain modes of transmission are exclusive to the newly whelped or neonates and also, because young dogs have not yet acquired immunity to parasites (Ramirez-Barrios et al., 2004). The infective stages of protozoan parasites are cysts and oocysts passed in the faeces and are capable of prolonged survival in the environmental. Infection and re-infection of human, domestic animals or wildlife can occur when the cysts or oocysts are ingested via contamination water, food materials or through host to host (Leonhard et al.,
2007). The clinical signs of protozoan infection are variable and occasionally some infected animals will be asymptomatic. However, severe clinical cases in young dogs will lead to diarrhea, anemia and death (RamirezBarrios et al., 2004). Previous studies have shown that intestinal protozoan parasites are common in dogs (Dubna et al., 2007; Mundim et al., 2007; OliveiraSequeira et al., 2002; Palmer et al., 2008; Papazahariadou et al., 2007; Ramirez-Barrios et al., 2004; Rimhanen-Finne et al., 2007). However, these studies were conducted in relatively limited geographic areas and resulted cannot necessarily be extrapolated to other regions. Understanding the epidemiology of the different parasites infections in a specific canine population is a useful tool for the veterinarian practitioner when he/she has to provide a clinical diagnostic (Ramirez-Barrios et al., 2004). Considering aspects related to public and animals' health, study of the prevalence of parasite infection in dogs should, therefore, be continuous task, with the most relevant aim being the establishment of control measures (Oliveira-Sequeira et al., 2002). The aim of our investigation was to determine the prevalence of protozoan infections in stray dogs in Kerman city, Iran. 


\section{MATERIALS AND METHODS}

Samples collected: Free roaming stray dogs were randomly selected among the animals that were euthanized in dog population control program (according to OIE Guidelines), which was organized by municipality. A total of 98 stray dogs faecal specimens were collected randomly from Kerman city of Iran, in 2009 , situated in the southern part of the national capital of Iran. The current population of Kerman is 2,652,413 (2006). The area is approximately $180726 \mathrm{~km}^{2}$ (the second largest prefecture in Iran). About 700,000 people live in the prefecture capital of Kerman, with the remainder dispersed over other 15 cities. Each faecal sample consisted of approximately $5 \mathrm{~g}$ of fresh stool, collected from rectum of the stray dogs and was accompanied by information about the gender and age of dogs. Then, the samples were immediately processed in the parasitology diagnostic laboratory of the Veterinary School of Shahid Bahonar University of Kerman.

Parasitological procedure: Faecal specimens were concentrated by the formalin-ether sedimentation method. Faecal smears of the sediment $(20 \mu \mathrm{L})$ were made and stained by the modified Ziehl-Neelson technique. The complete surface of the smear was examined for Cryptosporidium oocysts (Causape et al., 1996). Smear of the faeces was prepared and stained with trichrome and iodine Stain to detect cysts or trophozoites of Giardia and Entamoeba (Tanyuksel and Petri Jr, 2003). Also, samples were examined for the presence of Giardia cysts and trophozoites by centrifugal flotation in 33\% Zinc solphate solution (Mundim et al., 2007). Additionally, faecal flotation in Sheathers sugar solution $(500 \mathrm{~g}$ of sugar, $320 \mathrm{~mL}$ of water, $6.5 \mathrm{~g}$ of phenol), with a specific density of $1.3 \mathrm{~g} \mathrm{~mL}^{-1}$, were examined by light microscopy for Isospora spp. (Lindsay et al., 1997).
Analysis of results: The data analysis was performed separately by grouping the animals by age ( $\leq 1$ year and $>1$ year) and gender (male and female). In each case, the general prevalence for all intestinal protozoan parasites and the prevalence of each particular parasite were analyzed by using $\mathrm{x}^{2}$ test and 17 version of SPSS software.

\section{RESULTS}

Intestinal protozoan parasites were detected in feces of $13 \mathrm{dogs}$ (13.26\%). Four samples (4.08\%) contained Cryptosporidium spp. oocysts, 7 (7.14\%) contained Giardia spp. cysts and $5(5.1 \%)$ contained Isospora spp. oocysts (Table 1). Entamoeba spp. was not found in samples. Seven $(13.2 \%)$ of the female dogs were eliminating cysts or oocysts of intestinal protozoan in their faeces, while six $(13.3 \%)$ of the male dogs were infected. In this study, no statistical differences were found between infection by intestinal protozoan parasites and sex of the dogs $(\mathrm{p}>0.05)$. When the general prevalence analyzed by gender, no statistical differences in prevalence of Cryptosporidium, Giardia and Isoapora occurred between female dogs and male dogs $(\mathrm{p}>0.05)$. With respect to the age of the

Table 1: Prevalence of individual protozoan parasites in 98 dogs

\begin{tabular}{llll}
\hline Parasite & $\begin{array}{l}\text { No. of } \\
\text { infected dogs }\end{array}$ & $\begin{array}{l}\text { Relative } \\
\text { percentage }^{1}\end{array}$ & $\begin{array}{l}\text { Percentage of } \\
\text { total dogs }^{2}\end{array}$ \\
\hline Cryptosporidium & 4 & 30.77 & 4.80 \\
Giardia & 7 & 53.85 & 7.14 \\
Isospora & 5 & 38.46 & 5.10 \\
Entamoeba & 0 & 0.00 & 0.00 \\
Total $^{3}$ & 16 & &
\end{tabular}

${ }^{T}$ : Percentages were calculated as the number possessing an individual parasite divided by the total positive dogs $(13) ;{ }^{2}$ : Percentages were calculated as the number possessing 1,2 and 3 parasites species divided by the total dogs $(98){ }^{3}$ : This total is greater than 13 because of multiple parasitism

Table 2: Relationship of age and gender to prevalence of intestinal protozoan parasites in 98 dogs

\begin{tabular}{|c|c|c|c|c|c|c|c|c|}
\hline \multirow[b]{3}{*}{ Parasite } & \multicolumn{4}{|l|}{ Gender } & \multicolumn{4}{|l|}{ Age } \\
\hline & \multicolumn{2}{|l|}{ Female } & \multicolumn{2}{|l|}{ Male } & \multicolumn{2}{|l|}{$1 \geq 0$} & \multicolumn{2}{|l|}{$1<0$} \\
\hline & $\begin{array}{l}\text { No. of } \\
\text { infected } \\
\text { dogs }\end{array}$ & Percentage $^{1}$ & $\begin{array}{l}\text { No. of } \\
\text { infected } \\
\text { dogs }\end{array}$ & Percentage $^{1}$ & $\begin{array}{l}\text { No. of } \\
\text { infected } \\
\text { dogs }\end{array}$ & Percentage $^{1}$ & $\begin{array}{l}\text { No. of } \\
\text { infected } \\
\text { dog }\end{array}$ & Percentage $^{1}$ \\
\hline Cryptosporidium & 2 & 3.80 & 2 & 4.44 & 2 & 11.1 & 2 & 2.50 \\
\hline Giardia & 4 & 7.54 & 3 & 6.70 & 2 & 11.1 & 5 & 6.25 \\
\hline Isospora & 2 & 3.80 & 3 & 6.70 & 3 & $16.6^{*}$ & 2 & 2.50 \\
\hline Entamoeba & 0 & 0.00 & 0 & 0.00 & 0 & 0.0 & 0 & 0.00 \\
\hline Total parasitized dogs ${ }^{2}$ & 7 & 13.20 & 6 & 13.33 & 5 & $27.8^{*}$ & 8 & 10.00 \\
\hline Total $^{3}$ & 53 & & 45 & & 18 & & 80 & \\
\hline
\end{tabular}


dogs, five $(27.77 \%)$ of the puppies and eight $(10 \%)$ of the adults were infected by intestinal protozoan parasites. Statistical differences were found between infection by intestinal protozoan parasites and age of the dogs $(\mathrm{p}<0.05)$. When the general prevalence analyzed by age, statistical differences in prevalence of Isoapora occurred between $\operatorname{dogs} \leq 1$ years old and $1<\operatorname{dogs}(\mathrm{p}<0.05)$, but no statistical differences in prevalence of Cryptosporidium and Giardia occurred between this two groups ( $>00.05)$, (Table 2).

\section{DISCUSSION}

There have many studies of the general prevalence of intestinal protozoan parasites in dogs population worldwide (Dubna et al., 2007; Little et al., 2009; Mundim et al., 2007; Oliveira-Sequeira et al., 2002; Palmer et al., 2008; Papazahariadou et al., 2007; Ramirez-Barrios et al., 2004; Rimhanen-Finne et al., 2007). Prevalence is variable and depended on a number of factors including age, living conditions, diagnostic methodology employed and region studied (Mundim et al., 2007). In the present study, the overall prevalence of intestinal protozoan parasites in dogs was $13.26 \%$. The overall prevalence of Giardia infection (7.14\%) identified in this study is approximately similar to that previously reported in Australia (9.4\%) (Palmer et al., 2008), Greece (4.3\%) (Papazahariadou et al., 2007), Finland (5\%) (Rimhanen-Finne et al., 2007), United States (4\%) (Little et al., 2009). Although the overall prevalence of this parasite has been reported in Brazil (12.2\%) (Oliveira-Sequeira et al., 2002). Also Papini et al. (2005) and Szenasi et al. (2007) found high prevalence of infection of 55.2 and $58.8 \%$ in kenneled dogs respectively. It may be attributed to climate conditions. In this study, no statistical differences between infection by Giardia spp. and sex of the dogs were found. This confirms the finding of Kirkpatrick (1988); Bugg et al. (1999) and Huber et al. (2005). No statistical bias for Giardia infection due to age of dogs was found in the present study. This confirms the finding of Huber et al. (2005). The presence of Giardia spp. cysts was statistically associated with the presence of other intestinal protozoan. In the present study, Giardia spp. was mainly associated with Isospora spp. (3 cases) and this is in agreement with OliveiraSequeira et al. (2002), who found a greater prevalence of Giardia spp. associated with Isospora spp. than with other parasites.

With respect to Cryptosporidium spp., the infection rate of $4.08 \%$ is approximately in agreement with Papazahariadou et al. (2007), who found $2.8 \%$ of faecal samples from dogs collected in the Serres Prefecture,
Northern Greece to contain oocysts of Cryptosporidium spp. Epidemiological studies on the prevalence of Cryptosporidium in dogs showed the infection rates are variable according to geographic area and range from $1.4 \%$ in Czech (Dubna et al., 2007), 2.41\% in Brazil (Huber et al., 2005), 1.4\% Uberlandia (Mundim et al., 2007), 2\% in California (El-Ahraf et al., 1991). The likelihood of finding a source of oocyst could explain differences in prevalence between different areas. Other researchers suggested that prevalence may be highest in dogs from rural environments, since Cryptosporidiosis is primarily associated with farm livestock (Causape et al., 1996). Grimason et al. (1993) and coworker found $1 \%$ of the faecal specimen collected in seven public parks in Scotland contained Cryptosporidium oocysts, with a prevalence in individual parks ranging from $0-2.4 \%$. In this study, no statistical differences between infection with Cryptosporidium spp. and sex of the dogs were found. This confirms the finding of Huber et al. (2005). No statistical differences between infection with Cryptosporidium spp. and age of the dogs were found. Also, Causape et al. (1996) found no statistically differences in prevalence occurred between dogs under 1 year of age and dogs over 1 year old.

Another very common parasite found in the evaluated dogs was Isospora spp. (5.1\%), which shows that these coccidia are the main intestinal protozoa found in these pets, mostly in younger animals, as indicated by Ramirez-Barrios et al. (2004); Visco et al. (1977) and Vanparijs et al. (1991). In the presence study, we found statistical differences between infection by Isospora and age of the dogs $(\mathrm{p}<0.05)$. Isospora spp. was the most common enteric protozoan of stray dogs in our study (5.1\%), that similar results were obtained by Vanparijs et al. (1991), who observed Isospora spp. prevalence of 5.2\% in dogs in Belgium. Although, prevalence is lower than in previous surveys in Venezuela by Ramirez-Barrios et al. (2004), in Zaragoza, Spain by Causape et al. (1996) and in Sao Paulo State, Brazil by Oliveira-Sequeira et al. (2002), who found $8.1,9.9$ and $8.5 \%$ prevalence, respectively.

Frequency of intestinal protozoan parasites in the studied stray dogs was high. Giardia spp. and Isospora spp. were the most frequent parasites.

\section{CONCLUSION}

The results of this research showed that stray dogs are reservoirs for zoonotic intestinal protozoan parasites and should be considered important to public health. Stray dogs may have an important role in the transmission of some diseases and understanding the pathogenicity and epidemiology of potential zoonotic 
agents in this and other animals closely associated with human is fundamental to public health. So that, it is imperative for human to avoid faecal contamination in streets, public gardens and parks. Also stray dogs should be euthanized in dog population control program in Iran.

\section{REFERENCES}

Bugg, R.J., I.D. Robertson, A.D. Elliot and R.C.A. Thompson, 1999. Gastrointestinal parasites of urban dogs in Perth, Western Australia. The Vet. J., 157: 295-301. PMID: 10328840

Causape, A.C., J. Quilez, C. Sanchez-Acedo and E. Del Cacho, 1996. Prevalence of intestinal parasites, including Cryptosporidium parvum, in dogs in Zaragoza city, Spain. Vet. Parasitol., 67: 161-167. PMID: 9017864

Claerebout, E., S. Casaert, A.C. Dalemans, N. De Wilde and B. Levecke et al., 2009. Giardia and other intestinal parasites in different dog populations in Northern Belgium. Vet. Parasitol., 161: 41-46. PMID: 19155136

Dubna, S., I. Langrova, J. Napravnik, I. Jankovska and J. Vadlejch et al., 2007. The prevalence of intestinal parasites in dogs from Prague, rural areas and shelters of the Czech Republic. Vet. Parasitol., 145: 120-128. PMID: 17169492

El-Ahraf, A., J.V. Tacal Jr, M. Sobih, M. Amin and W. Lawrence et al., 1991. Prevalence of cryptosporidiosis in dogs and human beings in san bernardino county, California. J. Am. Vet. Med. Assoc., 198: 631-634. PMID: 2019531

Grimason, A.M., H.V. Smith, J.F.W. Parker, M.H. Jackson and P.G. Smith et al., 1993. Occurrence of Giardia sp. cysts and Cryptosporidium sp. Oocysts in faeces from public parks in the west of Scotland. Epidemiol. Infect., 110: 641-645. PMID: 8519329

Huber, F., T.C.B. Bomfim and R.S. Gomes, 2005. Comparison between natural infection by Cryptosporidium sp., Giardia sp. In dogs in two living situations in the West Zone of the municipality of Rio de Janeiro. Vet. Parasitol., 130: 69-72. PMID: 15893071

Kirkpatrick, C. E., 1988. Epizootiology of endoparasitic infections in pet dogs and cats presented to a veterinary teaching hospital. Vet. Parasitol., 30: 113-124. PMID: 3245104

Leonhard, S., K. Pfister, P. Beelitz, C. Wielinga and R.C.A. Thompson, 2007. The molecular characterization of Giardia from dogs in Southern Germany. Vet. Parasitol., 150: 33-38. PMID: 17913365
Lindsay, D.S., J.P. Dubey and B.L. Blagburn, 1997. Biology of Isospora spp. from humans, nonhuman primates and domestic animals. Clin. Microbiol. Rev., 10: 19-34. PMID: 8993857

Little, S.E., E.M. Johnson, D. Lewis, R.P. Jaklitsch and M.E. Payton et al., 2009. Prevalence of intestinal parasites in pet dogs in the United States. Vet. Parasitol., 166: 144-152. PMID: 19716659

Mundim, M.J.S., L.A.G. Rosa, S.M. Hortêncio, E.S.M. Faria and R.M. Rodr al., 2007. Prevalence of Giardia duodenalis and Cryptosporidium spp. In dogs from different living conditions in Uberlândia, Brazil. Vet. Parasitol., 144: 356-359. PMID: 17112668

Oliveira-Sequeira, T.C.G., A.F. Amarante, T.B. Ferrari and L.C. Nunes, 2002. Prevalence of intestinal parasites in dogs from Sao Paulo State, Brazil. Vet. Parasitol., 103: 19-27. PMID: 11750997

Palmer, C.S., R.C.A. Thompson, R.J. Traub, R. Rees and I.D. Robertson, 2008. National study of the gastrointestinal parasites of $\operatorname{dogs}$ and cats in Australia. Vet. Parasitol., 151: 181-190. PMID: 18055119

Papazahariadou, M., A. Founta, E. Papadopoulos, S. Chliounakis and K. Antoniadou-Sotiriadou et al., 2007. Gastrointestinal parasites of shepherd and hunting dogs in the Serres Prefecture, Northern Greece. Vet. Parasitol., 148: 170-173. PMID: 17573197

Papini, R., G. Gorini, A. Spaziani and G. Cardini, 2005. Survey on giardiosis in shelter dog populations. Vet. Parasitol., 128: 333-339. PMID: 15740871

Ramirez-Barrios, R.A., G. Barboza-Mena, J. Muoz, F. Angulo-Cubillan and E. Hernandez et al., 2004. Prevalence of intestinal parasites in dogs under veterinary care in Maracaibo, Venezuela. Vet. Parasitol., 121: 11-20. PMID: 15110399

Rimhanen-Finne, R., H.L. Enemark, J. Kolehmainen, P. Toropainen and M.L. Hanninen, 2007. Evaluation of immunofluorescence microscopy and enzyme-linked immunosorbent assay in detection of Cryptosporidium and Giardia infections in asymptomatic dogs. Vet. Parasitol., 145: 345-348. PMID: 17320291

Szenasi, Z., S. Marton, I. Kucsera, B. Tanczos and K. Horvath et al., 2007. Preliminary investigation of the prevalence and genotype distribution of Giardia intestinalis in dogs in Hungary. Parasitol. Res., 101: 145-152. DOI: 10.1007/s00436-007-0622-8

Tanyuksel, M. and W.A. Petri Jr., 2003. Laboratory diagnosis of amebiasis. Clin. Microbiol. Rev., 16: 713-729. DOI: $10.1128 / C M R .16 .4 .713-$ 729.2003 
Vanparijs, O., L. Hermans and L. Van Der Flaes, 1991. Helminth and protozoan parasites in dogs and cats in Belgium. Vet. Parasitol., 38: 67-73. PMID: 2024431
Visco, R.J., R. M. Corwin and L. A. Selby, 1977. Effect of age and sex on the prevalence of intestinal parasitism in dogs. J. Am. Vet. Med. Assoc., 170: 835-837. PMID: 856783 